\title{
Exploring Awareness of Breathing through Deep Touch Pressure
}

\author{
Annkatrin Jung \\ annjun@kth.se \\ KTH Royal Institute of Technology \\ Stockholm, Sweden
}

\author{
Miquel Alfaras \\ miquel.alfaras@uji.es \\ Universitat Jaume I \\ Castelló de la Plana, Spain
}

\author{
Pavel Karpashevich \\ pavelka@kth.se \\ KTH Royal Institute of Technology \\ Stockholm, Sweden
}

\author{
William Primett \\ wprimett@plux.info \\ FCT, Universidade NOVA de Lisboa \\ Lisbon, Portugal
}

Kristina Höök
khook@kth.se

KTH Royal Institute of Technology

Stockholm, Sweden

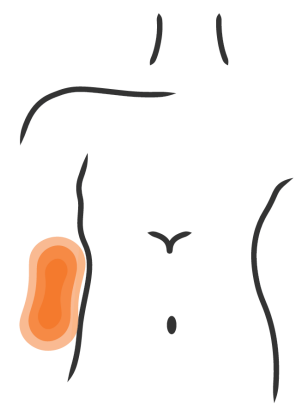

Figure 1: Deep pressure, pressing onto the torso

\begin{abstract}
Deep Pressure Therapy relies on exerting firm touch to help individuals with sensory sensitivity. We performed first-person explorations of deep pressure enabled by shape-changing actuation driven by breathing sensing. This revealed a novel design space with rich, evocative, aesthetically interesting interactions that can help increase breathing awareness and appreciation through: (1) applying symmetrical as well as asymmetrical pressure on the torso; (2) using pressure to direct attention to muscles or bone structure involved in different breathing patterns; (3) apply synchronous as well as asynchronous feedback following or opposing the user's breathing rhythm through applying rhythmic pressure. Taken together these explorations led us to design (4) breathing correspondence interactions - a balance point right between leading and following users' breathing patterns by first applying deep pressure - almost to the point of being unpleasant - and then releasing in rhythmic flow.
\end{abstract}

This work is licensed under a Creative Commons Attribution International 4.0 License.

CHI '21, May 8-13, 2021, Yokohama, Japan

(c) 2021 Copyright held by the owner/author(s)

ACM ISBN 978-1-4503-8096-6/21/05.

https://doi.org/10.1145/3411764.3445533

\section{CCS CONCEPTS}

- Human-centered computing $\rightarrow$ Haptic devices; Interaction design process and methods.

\section{KEYWORDS}

Deep Touch Pressure, Soma Design, Breathing, Wearables, ShapeChanging Interfaces

\section{ACM Reference Format:}

Annkatrin Jung, Miquel Alfaras, Pavel Karpashevich, William Primett, and Kristina Höök. 2021. Exploring Awareness of Breathing through Deep Touch Pressure. In CHI Conference on Human Factors in Computing Systems (CHI '21), May 8-13, 2021, Yokohama, Japan. ACM, New York, NY, USA, 15 pages. https://doi.org/10.1145/3411764.3445533

\section{INTRODUCTION}

Breathing feedback as well as shape-changing interactions are becoming increasingly popular topics in the field of interaction design. In the literature we find many examples of designing for breathing feedback in visual or aural form, or even, in some cases, using haptic feedback through different tangible media, such as shapechanging materials $[57,67]$. In parallel to this development, we note how there is plenty of technical work bringing forward new shape-changing materials [15], but fewer explorations of their aesthetic or functional affordances [71], calling for a more thorough understanding of the interactions between the artefact and those interacting with it [2]. 
Deep touch pressure (DTP) is a therapeutic method for treating sensory processing deficits [10]. The intention is to provide a calming effect through somatosensory sensation arising from hugging, cuddling, squeezing, stroking, or holding [43]. So far, DTP is relatively unexplored in interaction design research (with some notable exceptions [18, 22, 26, 27]). There are some commercial products in the shape of weighted blankets, garments, or inflatable vests [52]. However, there are few commercial products that rely on any interactive behaviour [53]. We saw an opportunity to use shape-changing elements that exert pressure on different body parts to achieve DTP, taking it as a starting point in our exploration of experiential aesthetic qualities of shape-changing pads connected to breathing sensing. By letting the shape-changing elements exert pressure on different locations on the torso, we explored new possibilities of interactive behaviours involving touch and breathing.

Our work builds on prior work combining biosensing and embodied exploration of actuation to focus on material properties [3, 78] and display bodily reactions as they unfold in real time [97]. The design process involved a series of collaborative design sessions engaging with couplings of breathing and pressure; construction of an experiential artefact [92] to explore the affordances of the socio-digital material [44]; as well as long-term first-person explorations of the impact of shape-changing deep pressure feedback coupled with conscious breathing practices. This was done in different teams: the first author of this paper spent a total of 5 months systematically exploring placement and interactive behaviours with two other authors as well as on her own, while the two remaining authors explored a slightly different version of the deep pressure material on and off during a period of one year.

Through our explorative design process, we uncovered potential for a number of novel design qualities that we will report on here: perceptual differences of applying deep pressure symmetrically and asymmetrically on the torso and how these may help to increase breathing awareness; opportunities for directing attention to different parts of the breathing apparatus, such as the bone structures or muscles involved, and thereby supporting learning a richer breathing repertory; the effects of providing feedback synchronously and asynchronously in harmony with, contrary to, or even out of sync with the user's breathing rhythm leading to a deeper aesthetic appreciation of one's breathing; as well as exploring the balance point between letting the system subtly lead or influence breathing patterns versus solely following the rhythm of the user's breathing in the interactions - an experience we will refer to as breathing correspondence. These explorations contribute to opening up the design space around shape-changing interfaces by characterizing potential experiential qualities and affordances based on felt bodily experiences of deep pressure for breathing awareness.

\section{BACKGROUND}

\subsection{Breathing Awareness}

A growing body of research in HCI focuses on designing interactive systems to extend breathing awareness. Prpa and colleagues [67] provide an overview of the underlying theoretical frameworks and design strategies used in breathing-based interactions. While some aim to trigger physiological responses related to alleviating stress and anxiety by slowing the breathing rate $[9,63,99]$, others utilise breathing patterns to promote mindfulness $[65,82]$ or to support communication between people through synchronisation of breathing [19, 42]. The somaesthetic design approach [44], which informed our work, utilises breathing as gentle guidance to develop sustained attention towards bodily sensations and learn to appreciate all the nuances of the felt experience of breathing $[68,90]$. By cultivating bodily and breathing awareness, users can come to better understand the connections between their physical and emotional experiences, thereby finding novel paths to regulate emotion and develop a higher sense of trust of their own body [8].

Many of these systems capture breathing data and translate it into sensory stimuli to externalise breathing, making it visually or tangibly accessible to users, creating immersive virtual and physical environments for engaging with breathing practices from mindfulness, yoga, Feldenkrais [58, 64, 82, 90, 102] and other body practices. While most interactions address auditory or visual modalities, there have been a few tangible designs which mirror or guide users' breathing processes, such as fidget spinners with added visual feedback [48], shape-changing airbags [105] or stuffed animals [4]. Other haptic systems mainly use vibration feedback [9, 20, 57]. While vibration feedback can be harder to follow than other types of feedback and is by some perceived as interruptive [28], it may also result in a greater subjective relaxation and decrease in breathing rate compared to, e.g., auditory feedback [9]. However, other forms of immediate haptic feedback on the torso have so far rarely been explored with the exception of a recent study by Foo et al. [26]. They noted that rhythmic pulsing compression applied on the torso showed potential to improve focused attention on breathing and adopt a slow breathing rhythm, making it an interesting option for further exploration.

\subsection{Shape-Changing Interfaces}

Shape changing interfaces constitute a novel interaction form as they enable interactive changes of the shape or texture of a material [2]. The increasing maturity of these shape-changing materials and tangible technologies has led to increased attention in the HCI and interaction design field. Recently, we have seen several attempts to clarify what this design space offers when building applications. For instance, Coelho and Zigelbaum [15] try to characterise technological properties of shape-changing materials, while Rasmussen and colleagues [71] identified eight different types of shape changes of relevance to different functional and hedonic design purposes. Others present particular design exemplars, including mobile devices [21, 30, 35], interactive tabletops [25, 94], furniture [32], toys [40], and interactive architecture [62]. A few shape-changing design exemplars have addressed breathing, such as a stuffed animal breathing in synchrony with its user [4], a photo frame reflecting a partner's breathing [42], or morphing physical environments for engaging with one's own breathing $[80,85]$. These systems mainly emphasise the visual aspect of shape-changing interaction $[42,58,80,85]$, and only in a few cases are they used to produce tactile feedback [105] or engaging with users' movements [96].

In comparison to the technical development, the aesthetic user experience of interacting with shape-changing materials as well as their affordances have not received as much attention $[2,71]$. 
Rasmussen and colleagues [71] devised two types of expressive parameters to characterise how movements of shape-changing interfaces are perceived by users. These include adjectives like smooth or angry as well as associations, such as a phone expressing sadness through a human-like sobbing pose. Due to their dynamic characteristics, users and designers often use metaphors to describe shape-changing behaviours, associating them with certain personality traits, animals or ascribing them other life-like qualities $[46,72]$.

\subsection{Deep Touch Pressure}

Deep touch pressure (DTP) is a method used in sensory integration therapy, aimed at treating sensory processing difficulties related to, amongst others, anxiety disorders, in particular anxiety experienced by those on the autism spectrum. [31, 36, 43]. The therapy involves using tools such as weighted garments and blankets to provide a comforting pressure sensation. Its calming effect can be attributed to increased activity of the parasympathetic nervous system, which plays a significant role in anxiety management [36]. In therapy practice, DTP has been applied to increase the ability to focus [24], reduce disruptive behaviour [69] and reduce anxiety symptoms [31] in patients with bipolar disorder, developmental disorders and those on the autism spectrum.

In HCI, different types of compression garments have been developed to provide DTP. Vaucelle et al. [101] designed a pressure vest containing pneumatic chambers. A more elaborate project is the Force Jacket [18], an upper body garment that uses pneumaticallyactuated airbags to create sensations of directly applied force and high frequency vibrations. Such vests are the most common form of designs for deep touch pressure and make up the majority of commercially available DTP products, such as the Tjacket [53] or the Squease vest [52]. Both are inflated using external pumps. Such vests have also been used to simulate hugs in long-distance interactions between parents and children [95]. An alternative type of pressure garment uses shape memory alloys which contract when heated, exerting pressure on the wearer's body [22]. Foo et al. [27] evaluated the user experience of compression garments based on shape memory alloys. They found that while individual preferences regarding different temporal patterns of pressure varied, it was overall experienced as more comfortable on the torso than on the shoulders or arms. Users reacted more sensitively towards pressure on the upper back than on the lower back, and felt that lower back compression supported their posture. The compression garments created a calming and warm effect, making participants feel secure and comforted.

The work we present here takes off from these earlier explorations, deepening prior work through exploring the aesthetic potential of deep pressure interactive shape-changing materials applied on and around the torso.

\section{DESIGN PROCESS}

Our design work started with a general interest in deep touch pressure, but when extrapolating from this concept we came to explore a range of interactions between physical pressure and breathing. Our explorations were done from an embodied, first-person perspective [37], putting the researchers themselves, their movements and subjective experiences at the centre of the design process. All five authors participated in this design process at different stages, in different constellations and with different variations of technological setups. Their demographic information is summarised in Table 1.

The process was initiated by the author Annkatrin via initial exploration of the breathing sensor, shape-changing actuators and fabrics for creating restriction and shape-change. This initial phase lasted for 1.5 months and allowed for discovering the affordances and experiential qualities of the available technology and materials, as well as experimenting with different shapes and sizes of the inflatable elements. Her first insights from this process informed possible interactions with different actuator sequences, which were further explored in a series of collaborative soma design workshops [44] performed by the authors Annkatrin, William and Miquel. These workshops are detailed in section 3.1. In total, four sessions took place over three weeks, iteratively exploring opportunities for connecting different actuation sequences and breathing techniques in relation to different areas of the body. The soma design workshops were followed by a continued systematic exploration of the deep pressure coupled with specific breathing techniques done by Annkatrin over a period of two months. This led to the construction of a wearable garment which places the shape-changing actuators on the upper and lower back to guide different ways of breathing, as shown in Figure 5. Annkatrin engaged with the garment daily over a period of three weeks (detailed in section 3.2). In parallel, two other authors (Pavel and Kia) explored a similar setup with identical shape-changing actuation and inflatable pillows encapsulated in the restrictive torso garment, but focused primarily on actuation. This design work took place over a one-year period and involved two somatic connoisseurs - a Feldenkrais expert and a professional singer - who helped to devise breathing techniques for the device and facilitated a richer understanding of the perceived bodily sensations of the interactions.

The results discussed in this paper are primarily based on the series of soma design workshops performed by Annkatrin, William and Miquel, as well as the further investigation of specific breathing techniques done by Annkatrin afterwards, with additional insights from the design work done by Pavel and Kia.

\subsection{Soma Design Sessions}

Over the course of four weeks, Annkatrin, William and Miquel performed a series of four collaborative soma design workshops exploring breathing-based interactions with shape-changing pressure feedback. The three authors are actively engaged in the field of embodied interaction with biofeedback systems, carrying modest experience with bodily practices such as yoga and Feldenkrais through regular practice both in and out of the lab. An additional participant, a graduate student from a product design background who is not among the authors of this paper, joined in the third session.

The workshop structure was grounded in soma design theory which places sensory experiences and appreciation at the centre of the design process to incorporate the soma, the integrated whole of body and mind, in a holistic manner and build a subjective understanding of one's somatic experiences [44]. Soma design methods focus on gaining awareness of physical experiences, exploring 
Table 1: Researchers' demographics (as of January 2021)

\begin{tabular}{|c|c|c|c|}
\hline Name & Gender & Age & Design and research background \\
\hline Annkatrin & Female & 23 & $\begin{array}{l}\text { M.Sc. in human-computer interaction and design, } 8 \\
\text { months experience with soma design }\end{array}$ \\
\hline Miquel & Male & 31 & $\begin{array}{l}\text { Graduate student in computer science, } 1 \text { year experience } \\
\text { with soma design }\end{array}$ \\
\hline Pavel & Male & 32 & $\begin{array}{l}\text { Graduate student in interaction design, } 3.5 \text { years experi- } \\
\text { ence with soma design }\end{array}$ \\
\hline William & Male & 24 & $\begin{array}{l}\text { Graduate student in sound-movement interaction, } 2 \\
\text { years experience with somatic practices }\end{array}$ \\
\hline Kia & Female & 56 & Professor in interaction design and expert in soma design \\
\hline
\end{tabular}

materials through embodied interaction, and testing out possible sensations first-hand, emphasizing a first-person, autobiographical perspective [37, 61].

Each of the four sessions lasted about three hours including the setup and incorporated shape-changing actuators in various shapes and sizes, shown in Figure 2, as well as additional fabrics to attach them to different parts of the body and create restriction. The actuators are part of the Soma Bits [13], a collection of shapes and actuators designed to facilitate soma design workshops, and consist of Thermoplastic Polyurethane (TPU)-coated nylon shapes which can be inflated or deflated at different speeds or stay at a constant level of inflation. Further in the text, we will be using "pillows", "inflatables", "pneumatic pads" interchangeably to describe these shapes. The actuation was controlled wirelessly with an Arduinobased device, capable of inflating and deflating the pneumatic pads at variable pump speed $(\max 3 \mathrm{~L} / \mathrm{min})$ within the pressure range of $450-1950 \mathrm{hPa}$, as well as providing real-time pressure sensor readings.

The framework used for sensing and actuation control is illustrated in Figure 3. To capture breathing, we used an inductive respiration (respiratory inductance plethysmography) sensor ${ }^{1}$ (1) which measures the relative displacement of the chest or stomach, depending on its placement. The sensor data is continuously acquired to compute the subject's breathing rate, which in turn influences the time dynamics of the shape-changing feedback. This data exchange is handled by (4) a Processing server, directing control messages between the browser-based Node-RED GUI (2) and

${ }^{1}$ PLUX RIP Sensor: https://biosignalsplux.com/products/sensors/respiration-inductive. html
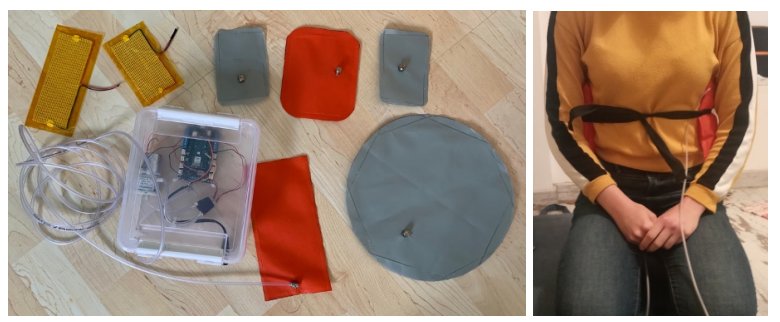

Figure 2: Embodied exploration of shape-changing actuators (left), worn e.g. on either side of the stomach (right) the actuation hardware ( $5 \&$ 6) via Open Sound Control (OSC) $)^{2}$. The visual interface allows for the different actuation sequences to be executed with adjustable parameters, while a separate Python script runs in the background for signal processing and feature extraction (3).

As soma design work incorporates bodily practices such as Feldenkrais and body scans to improve designers' somaesthetic awareness, each workshop began with a Feldenkrais practice to cultivate a sensitivity towards the bodily sensations and breathing, placing the somatic experiences in the foreground of the interaction with the materials. Afterwards, the participants took turns in placing the pads on different parts of their bodies to investigate how different placements, actuation sequences and body positions influence the experience. The explorations followed the soma design method of making strange [51], defamiliarizing habitual movements to draw attention towards subtle sensations and create a richer understanding of the experience.

In addition to taking notes, pictures and videos during the workshop, the participants used soma body sheets to document and visualise their subjective bodily experiences and share them with each other [50]. These sheets depict an empty outline of a human body onto which one can draw and write how they are experiencing different parts of their body, as shown in Figure 4. A reflection period amongst the participants concluded each session, using the drawings to initiate a discussion into how their bodily sensations had changed throughout the session.

These sessions enabled the authors to narrow down the physical schematics that would facilitate meaningful interactions. They included a set of suitable pad shapes/sizes and bodily placements that could be embedded into a wearable interactive system, later finalised by Annkatrin.

\subsection{Autobiographical Design: First-Person Engagement with Shape-Changing Materials and Breathing}

Based on the insights obtained during the soma design workshops, Annkatrin created a restrictive garment which contains the shapechanging pads, pressing against both the upper and lower back. It was inspired by the design of similar compression garments $[27,101]$, but not intended to represent a final design outcome

\footnotetext{
${ }^{2}$ Open Sound Control: http://opensoundcontrol.org
} 


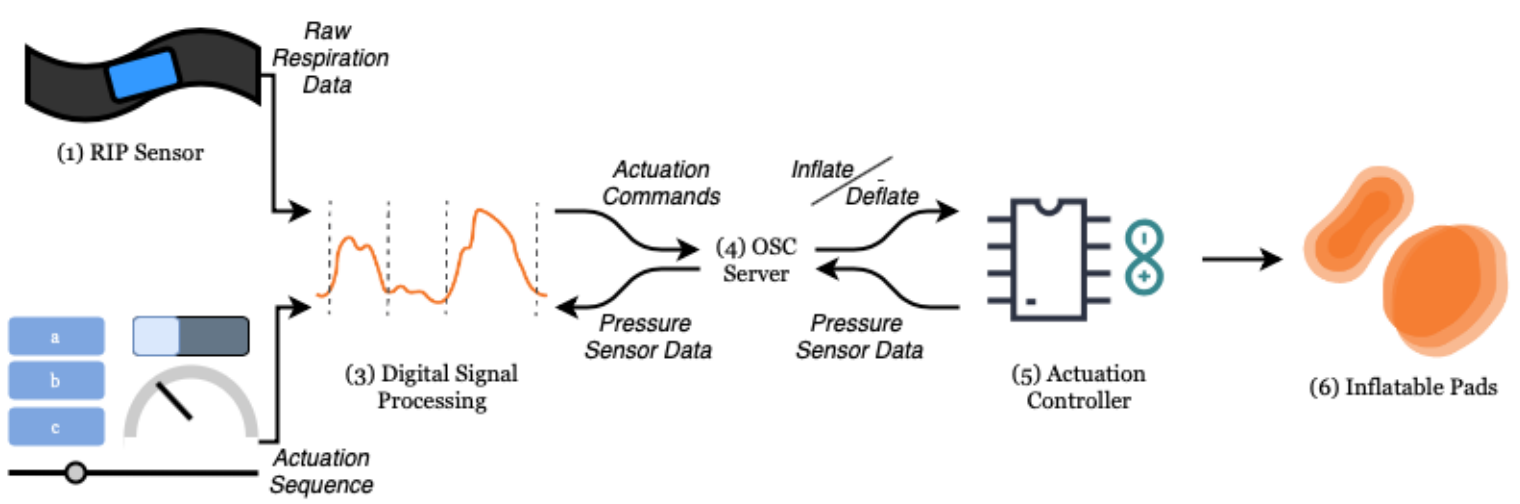

(2) Browser GUI

Figure 3: Modular framework for sensing and actuation control

nor a prototype for deep touch pressure therapy. Instead, the garment acted as an experiential artifact [92], an intermediate tool in the design process to present new and interesting experiences and discover their affordances through embodied interaction with breathing-based deep pressure.

The garment was sewn from a firm fabric and weighs $125 \mathrm{~g}$. It can be closed in the front with a loop-and-hook fastener which allows for a tight fit in the torso area, making the actuation clearly noticeable. While it is thus only suitable for users with a similar body shape, the loop strips allow for slight adjustments of the tightness. The pads can be placed in two pockets on the inside of the garment, one on the lower and one on the upper back. Figure 5 illustrates the approximate location of the pads when the garment is worn; however, we would like to note that the garment needs to be closed to apply deep pressure and is left open in Figure 5 to show the placement of the respiration sensor.

During the exploration, the actuator devices were placed externally and connected to the pads in the garment via two elastic air hoses that are laced through a small hole at the bottom of the lower pocket. Although this constrained Annkatrin's movements within a small area around the actuators and required her to be careful

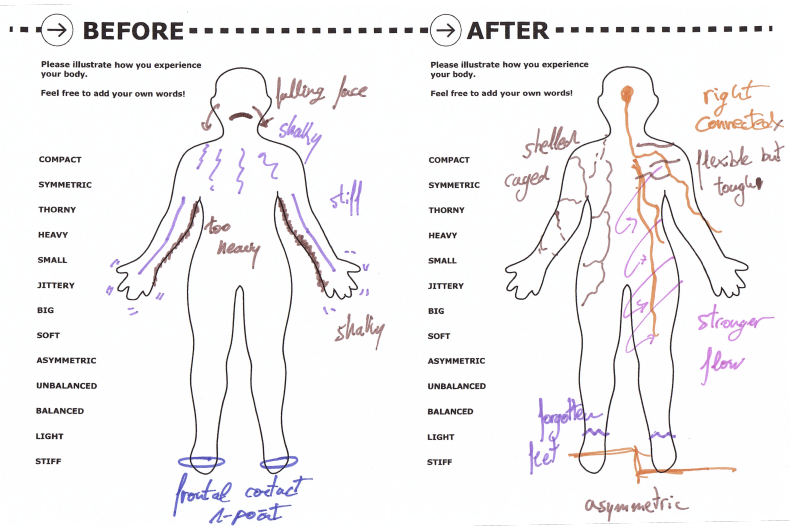

Figure 4: Example of a body sheet used to document the somatic experience during the design process when changing position, it did not detract from the interaction with the garment. The sound of the air pumps was dampened using noise-cancelling headphones playing white noise.

The garment was used along with an inductive respiration sensor to explore deep pressure feedback combined with different breathing techniques. The sensor was worn around the chest or stomach underneath the garment. Over the span of three weeks, Annkatrin conducted one to two sessions daily with a duration of 30-60 minutes each. These sessions were focused on exploring the potential impact of shape-changing deep pressure feedback coupled with a regular conscious breathing practice over a longer period of time on the subjective bodily experience and breathing awareness.

The sessions incorporated different breathing techniques as well as different contexts and positions, including lying on the back, sitting on a chair or doing other tasks such as reading or writing while wearing the garment. These positions and breathing patterns were informed by a two-months systematic exploration of deep pressure guiding different kinds of breath regulation, such as controlling the duration of inhalations and exhalations or alternating between diaphragmatic and thoracic breathing, done by Annkatrin in between the soma design workshops and the construction of the garment.

In total, four different breathing techniques were used:

(1) 5.5 pattern: Providing a constant rate of 5.5 inflate-deflate cycles, i.e. breaths, per minute, which has been associated with increased heart rate variability and relaxation [49]

(2) Three-part breath pattern: This was inspired by the threepart breathing technique from yoga [81] which combines diaphragmatic and thoracic breathing. One pad is placed on the lower and one on the upper back. The one on the lower back is inflated first followed by the one on the upper back, guiding the user to first fill the stomach with air and then let it expand into the rib cage and chest. Then, the pad on the upper back is deflated again followed by the one on the lower back, guiding the user to release the breath first from the chest and then the stomach. Each inflation and deflation interval has a duration of 2 seconds, with a 1 second pause in between breaths. 

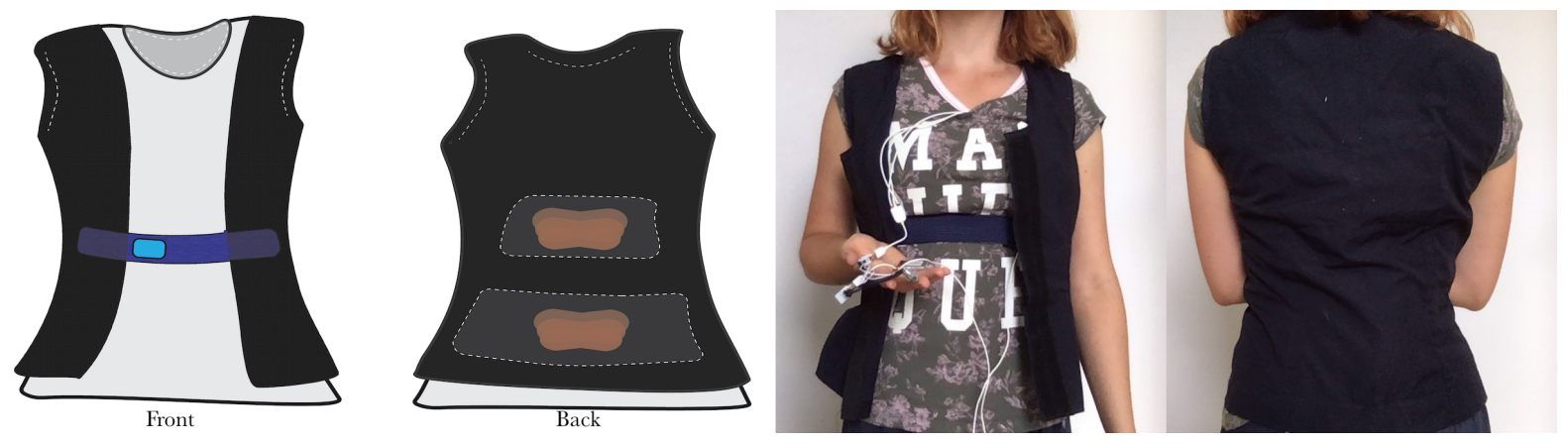

Figure 5: The garment, worn with a breathing sensor and one pad in each inner pocket

(3) Standardised feedback based on breathing sensor data with a 2 second pause in between breaths. It mirrors the user's breathing intervals, multiplied by 1.7 to gradually increase the duration of one breath without making the difference between two successive intervals too large.

(4) Adaptive feedback with an actuation change triggered by rapid breathing. At baseline, the inflation speed is set to $50 \%$ of the maximum, which makes the pads less noticeable. If the calculated inhale or exhale duration is shorter than 2 seconds, the inflation speed is increased to $100 \%$ until the breath becomes longer again.

At the beginning of each session, Annkatrin conducted a short body scan to take note of her present bodily sensations and breathing. Then, Annkatrin explored each combination of actuation pattern and position or context for at least 20 minutes before moving on to the next to experience more long-term effects of each condition. A single session was focused on a maximum of two different actuation patterns or two different body positions.

The exploration was documented in the form of an online diary as an intermediate step in the process of articulating the experiences. Initial impressions of the effects of the breathing exercises and the pressure feedback on the breathing and bodily experience as well as notable changes in the inductive respiration sensor data were noted down quickly and informally. As it was often difficult to articulate the primarily bodily explorations while still immersed in the process, it was necessary to take a short break afterwards before reflecting more deeply on the experiences based on the initial notes.

\section{EXPERIENTIAL AESTHETIC QUALITIES}

In the following, we present the four experiential aesthetic qualities that grew out of our design explorations. Such qualities bring out the aesthetic potential of interactions and how they are experienced in use [54], serving as an abstraction tool to articulate the insights which emerged over a series of explorations [88].

Each quality is introduced with a body of examples that highlight how the quality manifested in different first-person interactions. In reflection of these accounts and the data collected in the design explorations, we then outline the aesthetic potential of shapechanging deep pressure feedback and the overall influence this had on different breathing techniques. We discuss how pressure-based feedback can be used to cultivate breathing awareness, appreciation, controllability and deconstruction. The different experimental configurations allowed us to document a range of experiences relating to our breathing patterns, some yielding a greater awareness by reinforcing our habitual behaviour, and others disrupting the familiar movements.

\subsection{Placements on the Torso - Symmetric versus Asymmetric}

Insights of the importance of symmetrical as well as asymmetrical feedback arose from how the inflatable pads could be moved around to different locations on the torso. This made it possible to put deep pressure on, for example, the back right shoulder at the same time as another inflatable put pressure on the lower left-side of the belly. By symmetrical feedback, we are referring to pressure applied equally on the left and right sides of the torso, i.e. symmetrical on the lateral plane with the same actuation pattern. Asymmetrical feedback refers to one-sided pressure or pressure on both sides of the body, but a) on different body parts or b) with different actuation patterns.

4.1.1 Experiencing symmetric and asymmetric feedback. The team's explorations naturally began with symmetrical placements, leading to a whole range of interesting experiences. For example, when Annkatrin was experimenting with placing the inflatable pillows symmetrically on both shoulders, as shown in Figure 6, position (1), she experienced massage-like qualities or "someone putting a comforting hand on your shoulder". When placing the pads near the lower back or waist area when sitting or lying down, the pressure created a sense of support and stability. This was especially pronounced when placing the pads on either side of the lower back in a lying position, forming " a small indent in the floor to enclose my body, causing a comfortable and safe feeling." Annkatrin reported feeling unsteady and in lack of 'support' when the pillows were taken out or deflated, as if she would "roll to the left or right side at any moment". The symmetrical placement of the pads under the body was often helping Annkatrin to "feel more connected to the floor, more grounded in my body and my environment." Generally, interactions with symmetrical placements and patterns invoked calm and relaxed feelings: "I was left feeling much more in touch with my body, more calm and centred in myself. I felt loose, relaxed and a bit sleepy, similar to how I feel after a yog a practice or a good workout." 
Inspired by Feldenkrais exercises, Annkatrin, William and Miquel went on to experiment with asymmetrical actuation during the design workshops. Many Feldenkrais lessons aim to deconstruct the habitual coordination of movements. It is done in order to draw attention to their finer details or offer novel ways in which movement can be created, ultimately leading to alternative habitual movement patterns. Based on those Feldenkrais lessons, Annkatrin, William and Miquel tried to re-create a similar feeling of imbalance or deconstruction by placing the pillows on only one side of the body or putting two pillows simultaneously on different body parts. This created two different effects: while applying the actuation to two different body parts made it "hard for us to decide where to direct our attention which created an ill-fitting, incongruous experience", concentrating on only one side of the body "allowed us to become more aware of how we experienced that specific body part".

Later, Annkatrin continued to experiment with asymmetric actuation using two pads with conflicting or complementing rhythmic patterns at the same time. As opposed to random or conflicting feedback, we define complementing asymmetric feedback as requiring a regularity of sorts: the actuation intervals of one pad can be a multiple of the second pad, or one pad inflates while the other deflates. A conflicting pattern, on the other hand, has incoherent phases, switching from inflation to deflation at different times. These explorations revealed that "the placement of the pads had to be matched carefully to the actuation" to avoid interfering with each other or with the user's breathing. In particular, conflicting asymmetric actuation was perceived as distracting and uncomfortable, not leading to any deeper engagements. For example, placing the inflatables on the back of the thighs or using two very distinct actuation sequences "failed to evoke any strong effect". In some cases such placements could even lead to strong negative experiences, preventing learning. Placing the pads asymmetrically close to the neck, as shown in Figure 6, position (2), made Annkatrin feel "like I was not able to breathe properly", evoking associations with "having a snake wrapped around my throat". On the other hand, when the position and patterns were complementing - for example, when Annkatrin tried putting two pillows with one inflating twice as fast as the other on the lower back (Figure 6, position (3)) - they "created a very unusual twisting sensation in the torso". This in turn lead to insights on how locally applied effects can spread across the entire body, and helped Annkatrin to experience how different parts of the body are connected into a whole.

4.1.2 Reflecting on symmetric and asymmetric feedback. Symmetry is represented in many different forms in the HCI projects focused on breathing. In projects with visual feedback, symmetry has been expressed as: visualisation of two living lungs [1]; symmetrical geometrical figures [66, 99] or living virtual structures [64] located in the centre of the screen or virtual environment; or as abstract symmetrical visualisations taking the whole computer screen [59]. One can see symmetry expressed as varying light intensity in the Philips LivingColor pair of lights [20]. In the projects using vibration as output medium, symmetry is found in the relative position of the vibrating artefacts on the body, such as: centre of the belly [9]; centre of the upper chest [28]; or in the blanket that has the same vibro-tactile sensation on both sides of the body [20]. In projects using shape-changing actuation as a medium to display breathing,

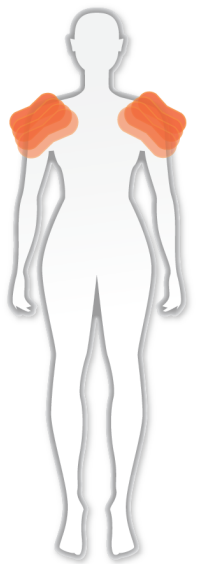

(1)

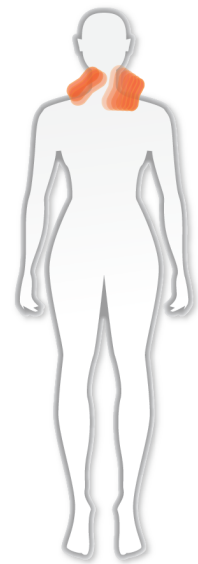

(2)

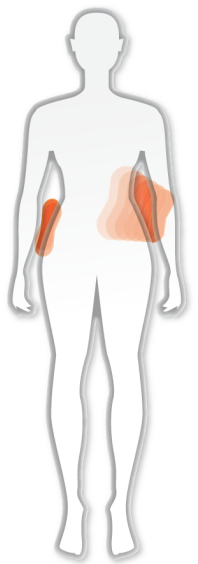

(3)
Figure 6: Applying symmetric and asymmetric deep pressure to the shoulders (1), neck (2) and lower back (3)

symmetry is found in: the equable expansion and contraction of a living wall [85]; in dynamic building structures [58, 80], as well as in the whole shape of a particular probe [4, 42]; or sometimes experienced by the whole body symmetrically [91].

Asymmetry as a design quality of breathing feedback has not been explored as much. There have only been a few asymmetrical representations, such as a visualisation of tides in the virtual garden [76], and a few examples of providing direct feedback onto only one side of the body, such as the guiding shape-changing pillow under the right palm of the hand in the works by Yu and colleagues [105].

The focus on symmetrical representations in design work may be rooted in the notion that symmetry is commonly associated with perceived beauty and satisfying aesthetic experience $[6,56]$. Well-balanced presentations of equal proportions are more compatible with classical mathematical structures, and the link between symmetry and beauty even has explicit associations with our biological presence [16]. However, absolute symmetry is not something we normally experience in the natural world, and leaves insufficient space for unpredictability and thereby discovery [56]. We also note that the idea that symmetry is aesthetically pleasing is more prevalent in western culture $[6,106]$

While we might perceive a human body as symmetrical when we compare our left half to our right half, it is obviously not. All the projects mentioned above in some way or other represent the lungs as a system with the perfect bilateral system. However, in a healthy individual, the left lung is slightly smaller than the right one to make some space for the heart - as the heart is slightly shifted to the left. Our limbs are also asymmetric, often making one side of our body the more dominant one. Some of us are writing with our right hands, some with the left, and even ambidextrous people find it hard to use both hands for every habitual task. Our limbs 
are not perfectly equal - there is often a limb length discrepancy. We are asymmetrical by nature and through training and everyday habituation, we might become even more asymmetrical in ways that harm us.

What then is the aesthetic potential of addressing these asymmetries in design? While symmetry and symmetrical feedback is a well-explored and articulated quality and approach, proven to be beneficial for creating feedback that is easy to grasp and follow, asymmetry has not been explored as much. What we found is also how asymmetrical feedback can be quite unpleasant and distracting. But when designed carefully, it can be very informative for the design process - as well as to users aiming to increase their breathing awareness. What is sometimes needed is a journey from asymmetry to putting those asymmetrical experiences back into a symmetric whole, as is often done in Feldenkrais exercises. For example, we might want to address the diagonal connection from the left lung into moving the hip on the right side, and then the connection from right lung to left hip movements, but then we need to put both together in order to explore and feel how all these parts are interconnected. If we leave the user with only one half of this experience, for example the connection from left lung to right hip, they might leave the exercise with a feeling of being lopsided.

Asymmetry as a quality is widely used in Feldenkrais practice for disrupting the processes of the familiar [104]. In Feldenkrais lessons it is achieved by directing attention to just one side of the body, one lung, particular limb or other body part. This allows us to break the circle of habitual experience and reconstruct them bit by bit, slowly and attentively into something new - evocative and unusual.

\subsection{Guiding Different Kinds of Breathing}

There are many possible breathing patterns promoted by different physical disciplines and body practices. Some are specifically targeted at focusing the mind and increase bodily awareness [11, 45]. To further explore the different muscles and bone structures involved in breathing, we decided to more deliberately impose deep pressure on different locations of the breathing apparatus in a manner that mirrors and encourages these different breathing patterns.

4.2.1 Experiencing breathing in different body parts. Applying deep pressure to different locations on the body not only made it possible to explore symmetric vs asymmetric feedback, but also to feel the breathing in different body parts. The actuation was adapted to the user's breathing by inflating the pillows during inhalation and deflating the pillows during exhalation, including a slight delay as the user's breathing intervals are computed from sensor data. This interaction is from here on referred to as following the user's breathing.

The explorations started with taking conscious breaths through the chest while placing the inflatable pillows on and under different body parts which revealed their potential to physically move the body in certain directions. This led to a range of interesting experiences that were not necessarily related to breathing, such as placing them under the feet to cause a movement which Annkatrin related to "walking on the spot."
However, Annkatrin experienced breathing-based actuation mirroring her breathing on the lower body as "uncomfortable and unnatural since I could not relate those body parts to my breathing", which made her feel "weird, like it's not supposed to be there". Instead, the pressure was more readily associated with breathing when it was applied close to the breathing apparatus, i.e. on the torso or upper body. Annkatrin associated forward and upward movements of the torso with an inhalation, "the pads seemed to push me to inhale", while moving backwards and pressing down on the torso were perceived as an exhalation. Placing the pads underneath each armpit "pushed my arms away from the body", creating a feeling of the chest expanding during an inhalation. In this way, the pressure was able to support certain ways of breathing by reinforcing the respective movements. Annkatrin found that when the pads were placed under the lower back, the pressure "encouraged me to breathe more with my stomach", while placing the pads on the upper back "made me breathe more with my chest." Guided by the pressure, Annkatrin was able to learn how to engage different muscles when inhaling and thus practice diaphragmatic or thoracic breathing.

To investigate these different breathing patterns further, Annkatrin, as well as Pavel and Kia in their explorations, created an actuation pattern based on the three-part breath, a technique from yoga practice which combines diaphragmatic and thoracic breathing in each inhalation and exhalation. Since this exercise requires a certain amount of awareness and control over which muscles are engaged when breathing, Annkatrin had to "focus a little more to direct my breath from my stomach to my chest and back." With one pad placed on the upper and another on the lower back however, she reported being able to follow the guidance of the deep pressure "without having to think about how I was supposed to breathe."

Over time, the exercises created a lasting effect which carried over into Annkatrin's everyday life: "I could feel the muscles in my torso more distinctively when walking around. I was experiencing my breathing more intensely throughout the day, leading me to take deeper breaths than before and use my stomach and chest more deliberately and distinctly while breathing.". This suggests that by applying deep pressure on certain body parts, the pads may help improve someone's control of their breathing over time, stimulating engagement with certain muscles to assist different ways of breathing with regular practice. While three-part breathing is just one possible option, a plethora of techniques exist, such as letting the rib cage expand towards the arms, chest, back or shoulders when inhaling. Each of these directions engages different muscles around the rib cage, that, when trained, can open up a rich repertory of breathing techniques.

4.2.2 Reflecting on different kinds of breathing. While many different muscles and bone structures are involved in breathing and enable distinct breathing patterns, breathing-based designs have mainly focused on reducing users' breathing rate [67]. Whilst some of these studies account for lung capacity (e.g [1]), attention towards individual breathing muscles is normally ignored, neglecting the opportunity to practice alternative breathing patterns using these muscles.

Only a few breathing-based designs employ different types of breathing, for example encouraging deep diaphragmatic/abdominal breathing to alleviate stress [66] and anxiety conditions [99]. Others 
measure breathing on different body parts [68], taking into account diaphragm expansion [19] as well as the rising and falling extent of the abdomen [80].

Outside of HCI, a handful of studies validate the use of additional sensor placements in order to differentiate between thoracic and abdominal breathing, such as the work by Hamnvik and colleagues on Yolo4Apne [33] which uses two inductive respiration sensors and [23] that uses three resistive stretch sensors across the torso. This approach has shown to be effective in extending measurement potentials, but it does not address alternative representations or interactions to guide users to explore novel breathing patterns.

So far, the interactive systems for breathing guidance do not reflect the immense resources we have for breathing, not only in regards to muscle engagement but also inhalation and exhalation sequences. Instead, breathing is often reduced to a single dimension that only operates from one part of the body, without registering these anatomical intricacies. What then are the technological interventions that may facilitate a richer breathing experience?

Though not extensive, three generic descriptors (clavicular, thoracic, and diaphragmatic [70]) provide a starting point to distinguish types of locational breathing. These also provide the foundation for the three-part breath technique (also known as Dirga Pranayama), which has been thoroughly studied in traditional yoga practice [81]. It demands specific attention to separate parts of the breathing apparatus, namely the abdomen (lower torso) and thorax (upper torso), and considers how these are used in conjunction to expand breathing capacity through full and complete breaths, compared to chest-based breathing.

However, there are many other breathing practices, such as those employed by professional singers or dancers, offering hitherto unexplored potential to provide engaging interactions. The way in which we breathe has a direct impact on the body's chemical makeup and behaviour of the autonomic nervous system [77]. Moreover, it is widely recognised that longer breaths incorporating the whole body can lead to improved overall well-being in the long term, particularly in regards to stress regulation [60]. Studies of alternative breathing patterns suggest that by engaging individual muscles and body structures, we can train ourselves to gradually gain greater awareness and control of our holistic body [98].

There exists a multitude of possibilities when it comes to engaging with breathing, and the same goes for halting the breathing. One can pull air into the lungs using the diaphragm or muscles in the neck, let the rib cage expand towards the back or the shoulders, and stop the breathing by pulling down the diaphragm, pushing the stomach out or tensing muscles around the rib cage. Many combinations of these techniques are possible, and can be further combined with other movements such as tensing or relaxing the pelvic floor. All add to the richness of the experience.

Thus, our explorations point towards a potential for interactions which incorporate a richer catalogue of breathing methods, creating the need to measure movements on different parts of the body to differentiate between small muscles involved in breathing. But the breathing rhythm does not consist of one-off breaths; instead, it is a pulsating, vibrant flow that engages a variety of muscles and body structures over time. This raises new questions as to how a system can turn a series of breaths into a malleable and adaptive rhythm, allowing the user to engage with their breathing pattern over an extended period of time.

\subsection{Synchronous vs Asynchronous Feedback}

By synchronous feedback we are referring to deep pressure that follows the user's breathing rhythm. Asynchronous feedback, on the other hand, provides a rhythm that is slightly or entirely out of phase with users' breathing, at times even pushing back right when the user inhales and expands their stomach or chest and vice-versa.

4.3.1 Experiencing synchronous and asynchronous feedback. At first, the explorations during the design workshops were focused on synchronous feedback, using actuation patterns which imitated the user's own breathing rhythm. These generally felt comfortable and supportive. The deep pressure drew attention to the breathing by making the breathing intervals more tangible and apparent, allowing Annkatrin to "appreciate my body and its ability to breathe steadily". The synchronous feedback also created an interesting interplay when sitting on a chair, since leaning back on the pads made the changes in pressure and thus the chest movements more intense, letting the entire body sway back and forth in the breathing rhythm. As the pads were physically pushing the body forwards to support inhalation, Annkatrin reported feeling like "they were in charge of my breathing and I could let myself surrender my control over my breathing to them", eliciting a sense of intimacy and safety.

Even when the actuation was not based on breathing sensor data but rather on pre-programmed sequences, Annkatrin, William and Miquel tried to match their breathing to the inflation and deflation of the pads. The pressure was not noticeable during the shift from deflation to inflation, which made Miquel feel "lost till I realised I should have been inhaling already", whereas Annkatrin felt "like I was not supposed or allowed to breathe" and thus held her breath until the pads began to inflate. Not being able to follow the rhythm set by the pads, which occurred for example when the intervals were very long, evoked feelings of guilt and frustration.

Pavel and Kia reported on similar experiences of frustration. Kia in particular found that for certain breathing exercises, she felt pressured to perform and when she could not adhere to the requirement, she felt as if the system was trying to discipline her. For example, she tested "square breathing" - a method where you are asked to breathe in while counting to five, hold your breath while counting to five, breathe out while counting to five and then hold your breath again, and then increase the counting, for example, up to 10 . The method is quite demanding. It helps train stamina and breathing ability for professional singers. The experience of being 'controlled' by the system became too strong for her when she did square breathing with the system.

Annkatrin, William and Miquel purposefully experimented with asynchronous patterns by using two inflatable pillows with conflicting patterns at the same time, inflating and deflating them in opposite or completely unrelated rhythms. Similar to asymmetric actuation, these patterns had to be crafted carefully to make sure that actuation sequences which were used simultaneously did not actively work against each other. Otherwise, they were dismissed as "hindering and annoying", such as when one pad was supporting inhalation by inflating while the other was discouraging it by 
deflating. At times, the asynchronicity also created unpleasant experiences, like feeling "dizzy or sea-sick" when using an asynchronous pattern which inflated one pad while deflating the other and vice versa while lying on the back.

Annkatrin noted that when the pads were contradicting each other or the natural flow of breathing, following their guidance felt like working against the body instead of engaging with the body. Switching the two pads during the three-part breath pattern, were guiding Annkatrin to first breathe in with the chest and then with the stomach. "This way of breathing made me feel like I was not getting enough air, thus appearing forced and unnatural." Annkatrin also reported finding it difficult and uncomfortable to breathe in an asynchronous rhythm, for example by holding her breath or taking intentionally short breaths. "Not following the pads seemed wrong since I was not engaging with my body but closing myself off to my body."

However, when all aspects complemented each other, the asynchronicity of the feedback was able to provoke interesting and unexpected experiences. The pads were generally seen as a "small extra pair of lungs", connecting inhalation to inflation and exhalation to deflation. To reverse this more familiar way of breathing, Annkatrin tried placing the pads on the stomach. This forced her to breathe in the opposite way, taking the pressure caused by the inflation as a cue to exhale and letting deflation make room for the stomach and chest to expand during inhalation. While such a breathing pattern accommodated the pressure on the stomach in a way that allowed Annkatrin to take deep breaths, it "required much more concentration because it was different from every other pattern I had tried so far". She had to figure out how to adapt to the pressure to be able to accommodate the pads and breathe comfortably, which made the pads seem like they were "restricting and controlling my breathing". Thus, the pressure forced Annkatrin to breathe very deliberately and thoughtfully, "drawing my attention to the mechanics of breathing and re-evaluating the pads' connection to my breathing". When used in this way, the asynchronous actuation was able to deconstruct breathing, creating the challenge to become comfortable with being thrown out of rhythm.

4.3.2 Reflecting on synchronous and asynchronous feedback. Synchrony is a quality that takes different forms in breathing-based design projects. The most straightforward is a direct mapping of a user's breathing pattern to certain feedback from the system. This is mostly done in phase, mapping expansion to inhalation and contraction to exhalation, with the exception of a photo frame that inflates when a remote person exhales and vice versa [42]. The inhalation has been mapped to many different output modalities, including the expansion of a visualised geometrical shape $[66,99,103]$ or pair of lungs [1], tides in a virtual environment [76], an increase in light intensity [20,90], as well as the expansion of a physical artefact $[4,42,58,85,91]$. Breathing phases can also be tied to the directions of movement of the protagonist in a video game [86] or of the user in a virtual environment $[17,66,99]$.

Synchrony may also involve more elaborate forms of feedback. When the user has fallen out of sync with the system, for example by breathing faster, the system can provide a guiding rhythm in form of vertically moving on-screen elements [60] or pulsating video and audio signals [29]. In multi-user systems, synchronous breathing of several users can make a sponge grow in a virtual environment [19] or trigger light effects on a garment worn by one of the users [79].

Asynchronous feedback can take form of shaping a soundscape based on different attributes of participants' breathing [102], as well as receiving instructions from a virtual breathing coach to modify the breathing rate without specifically addressing the user's actual breathing cycles ("continue breathing at a slower pace") [82] or showing the current breathing rate as a percentage of the individual resting breathing rate [59]. Asynchrony is also used in various negative feedback loops, in which a fast breathing rate increases the difficulty of a video game [63] or amusement ride [55], or lowers the quality of a song played in the background [34].

However, the majority of breathing-based systems reported in the literature encourages synchronous breathing patterns, aiming for synchronous feedback in harmony with the user's breathing rhythm. This is not surprising, as similar to symmetry, synchrony is an integral construct in technology, finance, molecular biology, physics, music and psychology which indicates coordination patterns among processes as well as precise coincidence of events in time [73]. Rhythm is one of the most important pillars of contemporary music, enabling the singers and musicians to not lose themselves in the music and stay in sync: one may miss a tone, but missing the rhythm can deteriorate the perception of the entire piece $[47,75]$. Nevertheless, a well-executed asynchronous element - syncopation, which is a deviation from an expected rhythmical pattern - enriches the piece and may bring listeners enjoyment and a desire to move [84]. Moving away from music, where tones are coherent and concurrently executed, language is also performed in antiphony as individuals take turns in conversations [74]. If we regard breathing as a dialogue between the system and the user, asynchronous forms of feedback can open up a new design space for deeper and more elaborate forms of interaction. Similar to asymmetry, careful and deft application of asynchronous elements in breathing feedback may break the patterns of habitual perception of breathing and make us more self-aware. We recognise that it sometimes takes time and effort to learn a difficult breathing practice, but still, it is important that the user does not lose faith in their ability along the way. There needs to be a path towards overcoming the uncomfortable experiences through practice, step by step learning more and more, but not demanding too much too soon.

\subsection{Breathing Correspondence: Finding a Balance between Leading and Following}

The findings made us think that a really interesting dialogue between the system and the user could be achieved, a dialogue that would rely on both letting users express their breathing, but at the same time being influenced, through feedback, encouragement or even strong pressure. We therefore continued to explore exactly how to find a balance point right between leading and following breathing patterns by first applying deep pressure - almost to the point of being unpleasant - and then releasing in rhythmic flow. We will refer to this experience as a breathing correspondence.

4.4.1 Experiencing different roles in the interaction. The explorations of different actuation patterns revealed how the inflatable pads could take on different roles in the interaction. If the actuation 
was following a predefined rhythm independent of the breathing sensor data, it was more likely to be perceived as not engaging in dialogue or adapting to the user, but instead leading the interaction and providing breathing instructions. They seemed to be "guiding or even steering the breath", reinforced by the pressure physically pushing the body forward.

When the actuation was based on the breathing sensor data, the pads assumed a more passive role in the interaction by following the user's breathing. In turn, this gave the user a more active role, allowing them to deliberately breathe in a different rhythm in an attempt to control or influence the pads. For example, during the design workshops Annkatrin, William and Miquel experimented with holding their breath and manipulating the length and depth of their inhalations and exhalations. Annkatrin reported having "the impression that the pads were listening to me which created a sense of intimacy and safety, like the pads were taking care of me". Such semi-autonomous interactions created an interesting contrast between breathing "with" the pads when following along to the actuation pattern and breathing "against" the pads when trying to change the pattern. However, it was not always clear whether the pad was adapting to breathing or vice versa, which introduced a sense of ambiguity in the interaction.

This potential for ambiguity motivated further attempts to combine both a leading and a following role in a single actuation pattern by extending the breathing feedback by different factors. We made it possible to influence the actuation by manipulating the breathing while the pressure was at the same time influencing the user to gradually take longer breaths. A dialogue between the system and the user emerged, using the pads as a communication channel: "As the pads were mirroring my breathing intervals while pushing me to gradually extend my breath, I was trying to match my breathing to the actuation, feeling the changes reflected by the pads."

The deep pressure becomes stronger with each interval almost to the point of being unpleasant, pushing Annkatrin to "take an excessively deep breath completely filling my lungs with air to the point of discomfort". Right before the pressure starts to become painful, it is released in a rhythmic flow, prompting the exhalation as a "sigh of relief". As the pressure intensifies and fades away gradually while providing a constant presence, it serves as a constant reminder to attend to the breath. The breathing feedback allowed Annkatrin to "become more aware of unintentional changes and try to make sense of them. For example, struggling to extend my breathing intervals made me wonder whether I was stressed or worried about something and therefore unable to take deep breaths." The pads were perceived as an extension of the physical self rather than an external influence, allowing Annkatrin to "engage in a dialogue with my body, not just the system itself."

However, when this balance between leading and following was disrupted, the pads began to dominate the interaction. After Annkatrin incorporated a threshold which increased the actuation speed and thus the pressure when the breathing intervals fell below two seconds, the experience changed significantly: "I was constantly aware of the pads and the pressure, trying to identify the current actuation speed and keep my breathing intervals above the threshold". Feeling the intervals become longer evoked a sense of accomplishment, while feeling them become shorter caused guilt and frustration which put Annkatrin under stress to extend her breathing. "It was a relief to take off the garment because I no longer felt pressured to actively control my breathing". Such exploration of the tipping point between leading and following can provide insights on how gradual pressure changes help to achieve a harmonic interaction, while strong or sudden changes are perceived like a firm command, taking over the control of the interaction.

Further explorations showed that just sitting with the pressure and breathing in one's own rhythm, neither trying to follow nor influence the actuation, could also create interesting experiences. In such interactions, the pads assumed the role of a companion which provided a "soothing sense of presence, like there was another person sitting next to me and sharing their breath with me". The pressure was not distracting, but rather seemed to complement one's individual breathing rhythm. Annkatrin reported that experiencing the pressure in the background while working led her to "take deeper breaths subconsciously without making an effort to do so".

Kia reported similar experiences - feeling that she could accept the system more willingly if she just let it act in the background while she was working away on her computer. Whether she was in fact following the feedback from the system or not became unimportant and she could relax into the experience.

4.4.2 Reflecting on establishing a breathing correspondence. According to their role in the interaction, breathing-based designs can be split into two distinct strands: in the Self - System - Self modality [67], the system is following the user's breathing pattern $[1,4,7,58,65,66,76,83,85,86]$, whereas in the System - Self System modality [67], the user is following the pattern provided by the system $[20,87,103,105]$. Looking at the prevalence of the two strands among HCI projects, the majority of breathing-based systems belong to the first group, providing a mere representation of the user's breathing pattern of the user and in the process taking the role of a passive follower. Only a few systems take a leading role in the whole dialogue $[87,103,105]$ or one of the interaction modalities $[9,64]$, providing users with a signal to follow. Despite the simplicity of the latter, it has proven to be a powerful interaction modality. Regardless of whether the user follows the interaction precisely or just leaves it as a peripheral stimulus, the interaction can provoke a relaxing and calming sensation when experienced for a prolonged period of time. This is also supported by the studies of Moraveji et al. $[59,60]$ showing the effectiveness of peripheral respiratory feedback on inducing a slower breathing rate without explicitly promoting focus on breath pacing or distracting users.

Some breathing-based systems make the distinction between leading and following more ambiguous by taking users' natural rhythm as a baseline, then feeding it back to them at a slightly slower pace to guide them to gradually extend their breathing [29, 60]. While this is a form of biofeedback aimed to achieve optimal HRV readings and supported by several studies [89, 93, 100], HRV can be misleading when misinterpreted or interpreted in a shallow sense [12].

The concept of correspondence proposed by Ingold [39] and further adapted and reworked into intimate correspondence by Höök et al. [38] calls for designing an interaction wherein the roles of leader and follower are unclear, the boundaries between user and system become dissolved and they start acting together as 'one'. This creates a more evocative communication to take form implicitly as 
there is no need to actively reply to the system's query - a correspondence relationship where users' breathing is simultaneously influencing and being influenced by the system.

\section{DISCUSSION AND CONCLUSION}

Through a first-person felt engagement over a longer time period, we uncovered several, previously unexplored breathing experiences spurred by different pressure patterns on the torso such as:

(1) imposing symmetrical and asymmetrical pressure as a path to draw attention towards different parts of the breathing apparatus, as well as spurring novel sensations by deconstructing habitual coordination of movements

(2) the sequencing of inflation/deflation in accordance with the user's engagement with different muscles when breathing, leading up to more intricate breathing practices

(3) providing synchronous or asynchronous feedback via rhythmic pressure that is in sync, slightly or entirely out of sync with users' breathing rhythm as a step towards deepening aesthetic appreciation and increasing awareness of possible breathing rhythms

(4) exploring the balance point between influencing and simply following the rhythm of the user's breathing - constituting a breathing correspondence experience where it is unclear whether the system or the user is driving the breathing rhythm

We want to emphasise that while our explorations were inspired by deep pressure therapy, the design space we opened also points to other experiences and needs beyond delivering calming and relaxing effects. Deepening users' appreciation of their own breathing can be an aesthetically interesting experience in itself. Breathing provides a rich inner universe where different body parts such as the lungs, rib cage, posture, bone structures and fascia are intricately connected in ways that can be more or less 'known' to us depending on how bodily aware we are. Different muscular tensions, engaging in habitual as well as non-habitual ways of breathing, can relate to or even spur emotional experiences in the short-term. Apart from the experience in the moment, the lingering effects after a session, feeling like you have had an 'inner' massage, as well as potential long-term lasting transfer effects into everyday life situations are particularly intriguing to explore further.

Apart from breathing guidance, pressure-based feedback may also be used on other parts of the body. By exploring experiences that breach into the domain of discomfort, we can begin to determine an appropriate equilibrium that lies between between gentle/subtle and uncomfortable/unpleasant [5]. We show how discomfort can be imposed by increased intensity of actuation, irregular and asymmetrical positioning of the pads, along with asynchronous inflation patterns that go against users' natural, unreflected breathing. When the interaction is engaging with the non-habitual, the system may elicit conscious physiological reactions, requiring users to be very much present with their breathing. However, if this intensity goes beyond a certain threshold, we lose this relationship and the experience becomes meaningless or even unsafe.

While we did not study social communication here, we know that learning to control our own breathing apparatus may also lead to better control over what we communicate to others. For example, consciously breathing in rhythm with someone else can lead to better communication and empathy [41]. Many body practices, such as improvisation dancing or martial arts, require coordinating breathing patterns not only with our own movements but also the person we are moving with [14].

Finally, we would like to point to how our first-person engagement turned out to be highly generative in terms of bringing out many design ideas for shape-changing materials applied on the torso. While more studies are needed to shift our subjective, personal understanding into reliable breathing engagements for larger user groups, our results show that first-person engagements will be one path to designing aesthetically rich, haptic engagements with shape-changing materials. This said, we are fully aware of the potential pitfalls of autobiographical design: the risk of designing only for the few people involved in the design process, ending up with designs that are irrelevant or even harmful to the targeted end-user group. The work we did here must be complemented with further design explorations, specifically aiming at different user groups, involving them in the design process. But as pointed out by Höök and colleagues [37] "this felt dimension, despite its subjective nature, is what provides rigour and structure to our design research". That is, the authenticity of the experiences we report on above relies on the long-term, deep, felt engagement of the designers - a rigour of a different ilk.

In summary, taken together, these first-person material explorations engaging with deep pressure feedback using shape-changing actuation managed to open a rich, somaesthetically evocative design space. We uncovered new paths of utilizing pressure-based feedback to cultivate appreciation, awareness, controllability and deconstruction of breathing, ultimately converging to an intimate relationship between system and user - a breathing correspondence.

\section{ACKNOWLEDGMENTS}

This work has been supported by AffecTech: Personal Technologies for Affective Health, Innovative Training Network funded by the H2020 People Programme under Marie Skłodowska-Curie grant agreement No 722022 and the Swedish Foundation for Strategic Research project RIT15-0046.

\section{REFERENCES}

[1] Ahmad Abushakra and Miad Faezipour. 2014. Augmenting Breath Regulation Using a Mobile Driven Virtual Reality Therapy Framework. IEEE fournal of Biomedical and Health Informatics 18, 3 (May 2014), 746-752. https://doi.org/ 10.1109/JBHI.2013.2281195 Conference Name: IEEE Journal of Biomedical and Health Informatics.

[2] Jason Alexander, Anne Roudaut, Jürgen Steimle, Kasper Hornbæk, Miguel Bruns Alonso, Sean Follmer, and Timothy Merritt. 2018. Grand Challenges in Shape-Changing Interface Research. In Proceedings of the $2018 \mathrm{CHI}$ Conference on Human Factors in Computing Systems (Montreal QC, Canada) (CHI 18). Association for Computing Machinery, New York, NY, USA, 1-14. https: //doi.org/10.1145/3173574.3173873

[3] Miquel Alfaras, Vasiliki Tsaknaki, Pedro Sanches, Charles Windlin, Muhammad Umair, Corina Sas, and Kristina Höök. 2020. From Biodata to Somadata. In Proceedings of the 2020 CHI Conference on Human Factors in Computing Systems. ACM, Honolulu HI USA, 1-14. https://doi.org/10.1145/3313831.3376684

[4] Ilhan Aslan, Hadrian Burkhardt, Julian Kraus, and Elisabeth André. 2016. Hold my Heart and Breathe with Me: Tangible Somaesthetic Designs. In Proceedings of the 9th Nordic Conference on Human-Computer Interaction - NordiCHI '16. ACM Press, Gothenburg, Sweden, 1-6. https://doi.org/10.1145/2971485.2996727

[5] Steve Benford, Chris Greenhalgh, Gabriella Giannachi, Brendan Walker, Joe Marshall, and Tom Rodden. 2012. Uncomfortable Interactions. In Proceedings of the SIGCHI Conference on Human Factors in Computing Systems (Austin, Texas, 
USA) (CHI '12). Association for Computing Machinery, New York, NY, USA, 2005-2014. https://doi.org/10.1145/2207676.2208347

[6] Marco Bertamini and Giulia Rampone. 2020. The Study of Symmetry in Empiri cal Aesthetics. In The Oxford Handbook of Empirical Aesthetics, Marcos Nada and Oshin Vartanian (Eds.). Oxford University Press, Oxford, United Kingdom. https://doi.org/10.1093/oxfordhb/9780198824350.013.23

[7] Peter M. Bingham, Jason H. T. Bates, John Thompson-Figueroa, and Thomas Lahiri. 2010. A Breath Biofeedback Computer Game for Children With Cystic Fibrosis. Clinical Pediatrics 49, 4 (April 2010), 337-342. https://doi.org/10.1177/ 0009922809348022

[8] Boris Bornemann, Beate M. Herbert, Wolf E. Mehling, and Tania Singer. 2015 Differential changes in self-reported aspects of interoceptive awareness through 3 months of contemplative training. Frontiers in Psychology 5 (Jan. 2015), 1-13. https://doi.org/10.3389/fpsyg.2014.01504

[9] Antoinette Leanna Bumatay and Jinsil Hwaryoung Seo. 2017. Investigating the Role of Biofeedback and Haptic Stimulation in Mobile Paced Breathing Tools. In Augmented Cognition. Neurocognition and Machine Learning - 11th International Conference, AC 2017, Held as Part of HCI International 2017, Vancouver, BC, Canada, Fuly 9-14, 2017, Proceedings, Part I (Lecture Notes in Computer Science, Vol. 10284), Dylan D. Schmorrow and Cali M. Fidopiastis (Eds.). Springer Vancouver, Canada, 287-303. https://doi.org/10.1007/978-3-319-58628-1_23

[10] Anita C. Bundy, Shelly Lane, Elizabeth A. Murray, and Anne G. Fisher. 2002 Sensory integration: theory and practice (2nd ed ed.). F.A. Davis, Philadelphia.

[11] Adam Burke and Sean Marconett. 2008. The Role of Breath in Yogic Traditions: Alternate Nostril Breathing. Biofeedback 36, 2 (2008), 67-69.

[12] Henning Cammann and Josef Michel. 2002. How to avoid misinterpretation of heart rate variability power spectra? Computer Methods and Programs in Biomedicine 68, 1 (2002), 15 - 23. https://doi.org/10.1016/S0169-2607(01)00154-7

[13] Charles Windlin, Anna Ståhl, Pedro Sanches, Vasiliki Tsaknaki, Pavel Karpashevich, Madeline Balaam, and Kristina Höök. 2019. Soma Bits - Mediating Technology to Orchestrate Bodily Experiences. Proceedings of the 4th Biennial Research Through Design Conference 4, Article 25 (March 2019), 1-16. https://doi.org/10.6084/

[14] Erwan Codrons, Nicolò F Bernardi, Matteo Vandoni, and Luciano Bernardi. 2014 Spontaneous group synchronization of movements and respiratory rhythms. PLoS One 9, 9 (2014), e107538.

[15] Marcelo Coelho and Jamie Zigelbaum. 2011. Shape-changing interfaces. Personal and Ubiquitous Computing 15, 2 (2011), 161-173. https://doi.org/10.1007/s00779010-0311-y

[16] Rodrigo Andrés Cárdenas and Lauren Julius Harris. 2006. Symmetrical decorations enhance the attractiveness of faces and abstract designs. Evolution and Human Behavior 27, 1 (Jan. 2006), 1-18. https://doi.org/10.1016/j.evolhumbehav. 2005.05.002

[17] Char Davies and John Harrison. 1996. Osmose: Towards Broadening the Aesthetics of Virtual Reality. SIGGRAPH Comput. Graph. 30, 4 (Nov. 1996), 25-28. https://doi.org/10.1145/240806.240808

[18] Alexandra Delazio, Ken Nakagaki, Roberta L. Klatzky, Scott E. Hudson, Jill Fain Lehman, and Alanson P. Sample. 2018. Force Jacket: Pneumatically-Actuated Jacket for Embodied Haptic Experiences. In Proceedings of the 2018 CHI Conference on Human Factors in Computing Systems (Montreal QC, Canada) (CHI '18). Association for Computing Machinery, New York, NY, USA, 1-12. https: //doi.org/10.1145/3173574.3173894

[19] John Desnoyers-Stewart, Ekaterina R. Stepanova, Philippe Pasquier, and Bern hard E. Riecke. 2019. JeL: Connecting Through Breath in Virtual Reality. In Extended Abstracts of the 2019 CHI Conference on Human Factors in Computing Systems (Glasgow, Scotland Uk) (CHI EA '19). Association for Computing Machinery, New York, NY, USA, 1-6. https://doi.org/10.1145/3290607.3312845

[20] Esko Dijk and Alina Weffers. 2011. Breathe with the Ocean. In Special Symposium at EuroHaptics 2010: Haptic and Audio-Visual Stimuli: Enhancing Experiences and Interaction. Universiteit Twente, Enschede, Amsterdam, Netherlands, 14.

[21] Panteleimon Dimitriadis and Jason Alexander. 2014. Evaluating the Effectiveness of Physical Shape-Change for in-Pocket Mobile Device Notifications. In Proceedings of the SIGCHI Conference on Human Factors in Computing Systems (Toronto, Ontario, Canada) (CHI '14). Association for Computing Machinery, New York, NY, USA, 2589-2592. https://doi.org/10.1145/2556288.2557164

[22] Julia C. Duvall, Nicholas Schleif, Lucy E. Dunne, and Brad Holschuh. 2019. Dynamic Compression Garments for Sensory Processing Disorder Treatment Using Integrated Active Materials. Journal of Medical Devices 13, 2 (June 2019), 021001. https://doi.org/10.1115/1.4042599

[23] Andreas Ejupi and Carlo Menon. 2018. Detection of Talking in Respiratory Signals: A Feasibility Study Using Machine Learning and Wearable Textile-Based Sensors. Sensors 18, 8 (Aug. 2018), 2474. https://doi.org/10.3390/s18082474 Number: 8 Publisher: Multidisciplinary Digital Publishing Institute.

[24] Doreen Fertel-Daly, Gary Bedell, and Jim Hinojosa. 2001. Effects of a weighted vest on attention to task and self-stimulatory behaviors in preschoolers with pervasive developmental disorders. American fournal of Occupational Therapy 55,6 (2001), 629-640.

[25] Sean Follmer, Daniel Leithinger, Alex Olwal, Akimitsu Hogge, and Hiroshi Ishii 2013. InFORM: Dynamic Physical Affordances and Constraints through Shape and Object Actuation. In Proceedings of the 26th Annual ACM Symposium on User Interface Software and Technology (St. Andrews, Scotland, United Kingdom) (UIST '13). Association for Computing Machinery, New York, NY, USA, 417-426. https://doi.org/10.1145/2501988.2502032

[26] Esther Foo, Justin Baker, Crystal Compton, and Brad Holschuh. 2020. Soft Robotic Compression Garment to Assist Novice Meditators. In Extended Abstracts of the 2020 CHI Conference on Human Factors in Computing Systems (Honolulu, HI, USA) (CHI EA '20). Association for Computing Machinery, New York, NY, USA, 1-8. https://doi.org/10.1145/3334480.3382919

[27] Esther W Foo, J. Walter Lee, Crystal Compton, Simon Ozbek, and Brad Holschuh. 2019. User experiences of garment-based dynamic compression for novel haptic applications. In Proceedings of the 23rd International Symposium on Wearable Computers - ISWC '19. ACM Press, London, United Kingdom, 54-59. https: //doi.org/10.1145/3341163.3347732

[28] Jérémy Frey, May Grabli, Ronit Slyper, and Jessica R. Cauchard. 2018. Breeze: Sharing Biofeedback through Wearable Technologies. In Proceedings of the 2018 CHI Conference on Human Factors in Computing Systems - CHI '18. ACM Press, Montreal QC, Canada, 1-12. https://doi.org/10.1145/3173574.3174219

[29] Asma Ghandeharioun and Rosalind Picard. 2017. BrightBeat: Effortlessly Influencing Breathing for Cultivating Calmness and Focus. In Proceedings of the 2017 CHI Conference Extended Abstracts on Human Factors in Computing Systems. Association for Computing Machinery, Denver, Colorado, USA, 1624-1631. https://doi.org/10.1145/3027063.3053164 Type: 10.1145/3027063.3053164.

[30] Antonio Gomes, Andrea Nesbitt, and Roel Vertegaal. 2013. MorePhone: A Study of Actuated Shape Deformations for Flexible Thin-Film Smartphone Notifications. In Proceedings of the SIGCHI Conference on Human Factors in Computing Systems (Paris, France) (CHI '13). Association for Computing Machinery, New York, NY, USA, 583-592. https://doi.org/10.1145/2470654.2470737

[31] Temple Grandin. 1992. Calming Effects of Deep Touch Pressure in Patients with Autistic Disorder, College Students, and Animals. Fournal of Child and Adolescent Psychopharmacology 2, 1 (Jan. 1992), 63-72. https://doi.org/10.1089/ cap.1992.2.63

[32] Erik Grönvall, Sofie Kinch, Marianne Graves Petersen, and Majken K. Rasmussen. 2014. Causing Commotion with a Shape-Changing Bench: Experiencing ShapeChanging Interfaces in Use. In Proceedings of the SIGCHI Conference on Human Factors in Computing Systems (Toronto, Ontario, Canada) (CHI '14). Association for Computing Machinery, New York, NY, USA, 2559-2568. https://doi.org/10. $1145 / 2556288.2557360$

[33] Sondre Hamnvik, Pierre Bernabé, and Sagar Sen. 2020. Yolo4Apnea: Realtime Detection of Obstructive Sleep Apnea. In Proceedings of the Twenty-Ninth International Joint Conference on Artificial Intelligence. International Joint Conferences on Artificial Intelligence Organization, Yokohama, Japan, 5234-5236. https://doi.org/10.24963/ijcai.2020/754

[34] Jason Harris, Sarah Vance, Odair Fernandes, Avinash Parnandi, and Ricardo Gutierrez-Osuna. 2014. Sonic Respiration: Controlling Respiration Rate through Auditory Biofeedback. In CHI '14 Extended Abstracts on Human Factors in Computing Systems (Toronto, Ontario, Canada) (CHI EA '14). Association for Computing Machinery, New York, NY, USA, 2383-2388. https://doi.org/10.1145/ 2559206.2581233

[35] Fabian Hemmert, Susann Hamann, Matthias Löwe, Anne Wohlauf, and Gesche Joost. 2010. Shape-Changing Mobiles: Tapering in One-Dimensional Deformational Displays in Mobile Phones. In Proceedings of the Fourth International Conference on Tangible, Embedded, and Embodied Interaction (Cambridge, Massachusetts, USA) (TEI '10). Association for Computing Machinery, New York, NY, USA, 249-252. https://doi.org/10.1145/1709886.1709936

[36] Hsin-Yung Chen, Hsiang Yang, Huang-Ju Chi, and Hsin-Ming Chen. 2013. Physiological Effects of Deep Touch Pressure on Anxiety Alleviation: The Weighted Blanket Approach. Journal of Medical and Biological Engineering 33, 5 (2013), 463. https://doi.org/10.5405/jmbe. 1043

[37] Kristina Höök, Baptiste Caramiaux, Cumhur Erkut, Jodi Forlizzi, Nassrin Hajinejad, Michael Haller, Caroline Hummels, Katherine Isbister, Martin Jonsson, George Khut, Lian Loke, Danielle Lottridge, Patrizia Marti, Edward Melcer, Florian Müller, Marianne Petersen, Thecla Schiphorst, Elena Segura, Anna Ståhl, Dag Svanæs, Jakob Tholander, and Helena Tobiasson. 2018. Embracing First-Person Perspectives in Soma-Based Design. Informatics 5, 1 (Feb. 2018), 8. https://doi.org/10.3390/informatics5010008

[38] Kristina Höök, Martin P. Jonsson, Anna Ståhl, and Johanna Mercurio. 2016. Somaesthetic Appreciation Design. In Proceedings of the 2016 CHI Conference on Human Factors in Computing Systems (CHI '16). Association for Computing Machinery, New York, NY, USA, 3131-3142. https://doi.org/10.1145/2858036. 2858583

[39] Tim Ingold. 2011. Being Alive: Essays on Movement, Knowledge and Description (1st edition ed.). Routledge, Abingdon, United Kingdom.

[40] Yuichiro Katsumoto, Satoru Tokuhisa, and Masa Inakage. 2013. Ninja Track: Design of Electronic Toy Variable in Shape and Flexibility. In Proceedings of the 7th International Conference on Tangible, Embedded and Embodied Interaction (Barcelona, Spain) (TEI '13). Association for Computing Machinery, New York, 
NY, USA, 17-24 https://doi.org/10.1145/2460625.2460628

[41] Peter E Keller, Giacomo Novembre, and Michael J Hove. 2014. Rhythm in joint action: psychological and neurophysiological mechanisms for real-time interpersonal coordination. Philosophical Transactions of the Royal Society B: Biological Sciences 369, 1658 (2014), 20130394.

[42] Jina Kim, Young-Woo Park, and Tek-Jin Nam. 2015. BreathingFrame: An In flatable Frame for Remote Breath Signal Sharing. In Proceedings of the Ninth International Conference on Tangible, Embedded, and Embodied Interaction (Stanford, California, USA) (TEI '15). Association for Computing Machinery, New York, NY, USA, 109-112. https://doi.org/10.1145/2677199.2680606

[43] Kirsten E. Krauss. 1987. The Effects of Deep Pressure Touch on Anxiety. American Journal of Occupational Therapy 41 (June 1987), 366-373. https: //doi.org/10.5014/ajot.41.6.366

[44] Kristina Höök. 2018. Designing with the Body: Somaesthetic Interaction Design The MIT Press, Cambridge, Massachusetts, United States.

[45] Sabina Kupershmidt and Traci Barnable. 2019. Definition of a Yoga Breathing (Pranayama) Protocol That Improves Lung Function. Holistic Nursing Practice 33, 4 (Aug. 2019), 197-203. https://doi.org/10.1097/HNP.0000000000000331

[46] Matthijs Kwak, Kasper Hornbæk, Panos Markopoulos, and Miguel Bruns Alonso. 2014. The design space of shape-changing interfaces: a repertory grid study. In Proceedings of the 2014 conference on Designing interactive systems - DIS '14. ACM Press, Vancouver, BC, Canada, 181-190. https://doi.org/10.1145/2598510. 2598573

[47] Daniel J. Levitin. 2019. This is your brain on music: understanding a human obsession. Penguin Books, London.

[48] Rong-Hao Liang, Bin Yu, Mengru Xue, Jun Hu, and Loe M. G. Feijs. 2018 BioFidget: Biofeedback for Respiration Training Using an Augmented Fidget Spinner. In Proceedings of the 2018 CHI Conference on Human Factors in Computing Systems - CHI '18. ACM Press, Montreal QC, Canada, 1-12. https://doi.org/10.1145/3173574.3174187

[49] I-Mei Lin, L.Y. Tai, and Sheng-Yu Fan. 2014. Breathing at a rate of 5.5breaths per minute with equal inhalation-to-exhalation ratio increases heart rate variability. International fournal of Psychophysiology 91, 3 (2014), 206 - 211. https://doi. org/10.1016/j.ijpsycho.2013.12.006

[50] Lian Loke and George Poonkhin Khut. 2014. Intimate aesthetics and facilitated interaction. In Interactive Experience in the Digital Age. Springer, Sydney, Australia, 91-108.

[51] Lian Loke and Toni Robertson. 2013. Moving and making strange: An em bodied approach to movement-based interaction design. ACM Transactions on Computer-Human Interaction 20, 1 (March 2013), 1-25. https://doi.org/10.1145/ 2442106.2442113

[52] Squease Ltd. 2014. Squease Vest. Squease Ltd. https://www.squeasewear.com

[53] Tware Pte Ltd. 2020. Tjacket. Tware Pte Ltd. https://www.mytjacket.com

[54] Jonas Löwgren. 2009. Toward an articulation of interaction esthetics. The New Review of Hypermedia and Multimedia 15 (08 2009), 129-146. https://doi.org/10. 1080/13614560903117822

[55] Joe Marshall, Duncan Rowland, Stefan Rennick Egglestone, Steve Benford, Brendan Walker, and Derek McAuley. 2011. Breath Control of Amusement Rides. In Proceedings of the SIGCHI Conference on Human Factors in Computing Systems (Vancouver, BC, Canada) (CHI '11). Association for Computing Machinery, New York, NY, USA, 73-82. https://doi.org/10.1145/1978942.1978955

[56] Ian Christopher McManus. 2005. Symmetry and asymmetry in aesthetics and the arts. European Review 13, S2 (Oct. 2005), 157-180. https://doi.org/10.1017/ S1062798705000736

[57] Pardis Miri, Robert Flory, Andero Uusberg, Heather Culbertson, Richard H. Harvey, Agata Kelman, Davis Erik Peper, James J. Gross, Katherine Isbister, and Keith Marzullo. 2020. PIV: Placement, Pattern, and Personalization of an Inconspicuous Vibrotactile Breathing Pacer. ACM Trans. Comput.-Hum. Interact. 27, 1, Article 5 (Jan. 2020), 44 pages. https://doi.org/10.1145/3365107

[58] Stuart Moran, Nils Jäger, Holger Schnädelbach, and Kevin Glover. 2016. ExoPranayama: a biofeedback-driven actuated environment for supporting yoga breathing practices. Pers. Ubiquitous Comput. 20, 2 (2016), 261-275. https: //doi.org/10.1007/s00779-016-0910-3

[59] Neema Moraveji, Athman Adiseshan, and Takehiro Hagiwara. 2012. BreathTray: Augmenting Respiration Self-Regulation without Cognitive Deficit. In CHI '12 Extended Abstracts on Human Factors in Computing Systems (Austin, Texas, USA) (CHI EA '12). Association for Computing Machinery, New York, NY, USA, 2405-2410. https://doi.org/10.1145/2212776.2223810

[60] Neema Moraveji, Ben Olson, Truc Nguyen, Mahmoud Saadat, Yaser Khalighi, Roy Pea, and Jeffrey Heer. 2011. Peripheral paced respiration: influencing user physiology during information work. In Proceedings of the 24th annual ACM symposium on User interface software and technology (UIST '11). Association for Computing Machinery, New York, NY, USA, 423-428. https://doi.org/10.1145/ 2047196.2047250

[61] Carman Neustaedter and Phoebe Sengers. 2012. Autobiographical Design in HCI Research: Designing and Learning through Use-It-Yourself. In Proceedings of the Designing Interactive Systems Conference (Newcastle Upon Tyne, United Kingdom) (DIS '12). Association for Computing Machinery, New York, NY, USA
514-523. https://doi.org/10.1145/2317956.2318034

[62] Kas Oosterhuis and Nimish Biloria. 2008. Interactions with Proactive Architectural Spaces: The Muscle Projects. Commun. ACM 51, 6 (June 2008), 70-78. https://doi.org/10.1145/1349026.1349041

[63] Avinash Parnandi, Beena Ahmed, Eva Shipp, and Ricardo Gutierrez-Osuna. 2014. Chill-Out: Relaxation Training through Respiratory Biofeedback in a Mobile Casual Game. In Mobile Computing, Applications, and Services, Gérard Memmi and Ulf Blanke (Eds.). Vol. 130. Springer International Publishing, Cham, 252-260. https://doi.org/10.1007/978-3-319-05452-0_18 Series Title: Lecture Notes of the Institute for Computer Sciences, Social Informatics and Telecommunications Engineering.

[64] Rakesh Patibanda, Florian 'Floyd' Mueller, Matevz Leskovsek, and Jonathan Duckworth. 2017. Life Tree: Understanding the Design of Breathing Exercise Games. In Proceedings of the Annual Symposium on Computer-Human Interaction in Play (Amsterdam, The Netherlands) (CHI PLAY '17). Association for Computing Machinery, New York, NY, USA, 19-31. https://doi.org/10.1145/3116595. 3116621

[65] Andrea M. Pisa, George Chernyshov, Andriana F. Nassou, and Kai Kunze. 2017. Towards Interactive Mindfulness Training Using Breathing Based Feedback. In Proceedings of the 2017 ACM International foint Conference on Pervasive and Ubiquitous Computing and Proceedings of the 2017 ACM International Symposium on Wearable Computers (Maui, Hawaii) (UbiComp '17). Association for Computing Machinery, New York, NY, USA, 688-692. https: //doi.org/10.1145/3123024.3129268

[66] Mirjana Prpa, Karen Cochrane, and Bernhard E Riecke. 2016. Hacking Alternatives in 21st Century: Designing a Bio-Responsive Virtual Environment for Stress Reduction. In Pervasive Computing Paradigms for Mental Health: 5th International Conference, MindCare 2015, Milan, Italy, September 24-25, 2015, Revised Selected Papers, Vol. 604. Springer, Milan, Italy, 34.

[67] Mirjana Prpa, Ekaterina R. Stepanova, Thecla Schiphorst, Bernhard E. Riecke, and Philippe Pasquier. 2020. Inhaling and Exhaling: How Technologies Can Perceptually Extend our Breath Awareness. In Proceedings of the $2020 \mathrm{CHI}$ Conference on Human Factors in Computing Systems. ACM, Honolulu HI USA, 1-15. https://doi.org/10.1145/3313831.3376183

[68] Mirjana Prpa, Kıvanç Tatar, Jules Françoise, Bernhard Riecke, Thecla Schiphorst, and Philippe Pasquier. 2018. Attending to Breath: Exploring How the Cues in a Virtual Environment Guide the Attention to Breath and Shape the Quality of Experience to Support Mindfulness. In Proceedings of the 2018 on Designing Interactive Systems Conference 2018 - DIS '18. ACM Press, Hong Kong, China, 71-84. https://doi.org/10.1145/3196709.3196765

[69] Shawn P. Quigley, Lloyd Peterson, Jessica E. Frieder, and Stephanie Peterson. 2011. Effects of a weighted vest on problem behaviors during functional analyses in children with Pervasive Developmental Disorders. Research in Autism Spectrum Disorders 5 (2011), 529-538.

[70] Swami Rama, Rudolph Ballentine, and Alan Hymes. 1998. Science of Breath: A Practical Guide. Himalayan Institute Press, Honesdale, Pennsylvania, United States. https://books.google.de/books?id=oSg0N_GrRsQC

[71] Majken K. Rasmussen, Esben W. Pedersen, Marianne G. Petersen, and Kasper Hornbæk. 2012. Shape-Changing Interfaces: A Review of the Design Space and Open Research Questions. In Proceedings of the SIGCHI Conference on Human Factors in Computing Systems (Austin, Texas, USA) (CHI '12). Association for Computing Machinery, New York, NY, USA, 735-744. https://doi.org/10.1145/ 2207676.2207781

[72] Majken K. Rasmussen, Giovanni M. Troiano, Marianne G. Petersen, Jakob G. Simonsen, and Kasper Hornbæk. 2016. Sketching Shape-Changing Interfaces: Exploring Vocabulary, Metaphors Use, and Affordances. In Proceedings of the 2016 CHI Conference on Human Factors in Computing Systems (San Jose, California, USA) (CHI '16). Association for Computing Machinery, New York, NY, USA, 2740-2751. https://doi.org/10.1145/2858036.2858183

[73] Andrea Ravignani. 2017. Interdisciplinary debate: Agree on definitions of synchrony. Nature 545 (05 2017), 158-158. https://doi.org/10.1038/545158c

[74] Andrea Ravignani, Daniel L Bowling, and W Tecumseh Fitch. 2014. Chorusing, synchrony, and the evolutionary functions of rhythm. Frontiers in Psychology 5 (2014), 1-15.

[75] Steve Reich. 2004. Writings on Music 1965-2000: 1965-2000. Oxford University Press, USA. https://doi.org/10.1093/acprof:oso/9780195151152.001.0001

[76] Joan Sol Roo, Renaud Gervais, Jeremy Frey, and Martin Hachet. 2017. Inner Garden: Connecting Inner States to a Mixed Reality Sandbox for Mindfulness. In Proceedings of the 2017 CHI Conference on Human Factors in Computing Systems (Denver, Colorado, USA) (CHI '17). Association for Computing Machinery, New York, NY, USA, 1459-1470. https://doi.org/10.1145/3025453.3025743

[77] Marc A. Russo, Danielle M. Santarelli, and Dean O'Rourke. 2017. The physiological effects of slow breathing in the healthy human. Breathe 13, 4 (Dec. 2017), 298-309. https://doi.org/10.1183/20734735.009817

[78] Pedro Sanches, Kristina Höök, Corina Sas, and Anna Ståhl. 2019. Ambiguity as a Resource to Inform Proto-Practices: The Case of Skin Conductance. ACM Transactions on Computer-Human Interaction 26, 4 (July 2019), 1-32. https: //doi.org/10.1145/3318143 
[79] Thecla Schiphorst. 2006. Breath, skin and clothing: Using wearable technologies as an interface into ourselves. International fournal of Performance Arts and Digital Media 2, 2 (2006), 171-186. https://doi.org/10.1386/padm.2.2.171_1

[80] Holger Schnädelbach, Ainojie Irune, David Kirk, Kevin Glover, and Patrick Brundell. 2012. ExoBuilding: Physiologically Driven Adaptive Architecture. ACM Trans. Comput.-Hum. Interact. 19, 4, Article 25 (Dec. 2012), 22 pages. https: //doi.org/10.1145/2395131.2395132

[81] Pallav Sengupta. 2012. Health Impacts of Yoga and Pranayama: A State-of-theArt Review. International journal of preventive medicine 3 (07 2012), 444-58.

[82] Ameneh Shamekhi and Timothy Bickmore. 2018. Breathe Deep: A Breath Sensitive Interactive Meditation Coach. In Proceedings of the 12th EAI International Conference on Pervasive Computing Technologies for Healthcare (New York, NY, USA) (PervasiveHealth '18). Association for Computing Machinery, New York, NY, USA, 108-117. https://doi.org/10.1145/3240925.3240940

[83] Chris D Shaw, Diane Gromala, and A Fleming Seay. 2007. The Meditation Chamber: Enacting Autonomic Senses. In 4th International Conference on Enactive Interfaces 2007. Association ACROE, Grenoble, France, 405-424.

[84] George Sioros, Marius Miron, Matthew Davies, Fabien Gouyon, and Guy Madison. 2014. Syncopation creates the sensation of groove in synthesized music examples. Frontiers in Psychology 5 (2014), 1036.

[85] Heikki Sjöman, Nazare Soares, Martinus Suijkerbuijk, Jørgen Blindheim, Martin Steinert, and Dag T. Wisland. 2018. The Breathing Room: Breathing Interval and Heart Rate Capturing through Ultra Low Power Radar. In Extended Abstracts of the 2018 CHI Conference on Human Factors in Computing Systems (Montreal QC Canada) (CHI EA '18). Association for Computing Machinery, New York, NY, USA, 1-4. https://doi.org/10.1145/3170427.3186473

[86] Tobias Sonne and Mads Møller Jensen. 2016. ChillFish: A Respiration Game for Children with ADHD. In Proceedings of the TEI '16: Tenth International Conference on Tangible, Embedded, and Embodied Interaction (Eindhoven, Netherlands) (TEI '16). Association for Computing Machinery, New York, NY, USA, 271-278. https://doi.org/10.1145/2839462.2839480

[87] Florian Soyka, Markus Leyrer, Joe Smallwood, Chris Ferguson, Bernhard E. Riecke, and Betty J. Mohler. 2016. Enhancing stress management techniques using virtual reality. In Proceedings of the ACM Symposium on Applied Perception (SAP '16). Association for Computing Machinery, Anaheim, California, 85-88. https://doi.org/10.1145/2931002.2931017

[88] Anna Ståhl, Jonas Löwgren, and Kristina Höök. 2014. Evocative Balance Designing for Interactional Empowerment. International Journal of Design 8, 1 (2014), 43-57. QC 20141110.

[89] Patrick R. Steffen, Tara Austin, Andrea DeBarros, and Tracy Brown. 2017-0825. The Impact of Resonance Frequency Breathing on Measures of Heart Rate Variability, Blood Pressure, and Mood. Frontiers in Public Health 5 (2017-08-25), 1-6. https://doi.org/10.3389/fpubh.2017.00222

[90] Anna Ståhl, Martin Jonsson, Johanna Mercurio, Anna Karlsson, Kristina Höök, and Eva-Carin Banka Johnson. 2016. The Soma Mat and Breathing Light. In Proceedings of the 2016 CHI Conference Extended Abstracts on Human Factors in Computing Systems - CHI EA '16. ACM Press, San Jose, California, USA, 305-308. https://doi.org/10.1145/2851581.2889464

[91] Xiaotian Sun and Kiyoshi Tomimatsu. 2017. Breath is to be perceived - breathing signal sharing involved in remote emotional communication. In Distributed Ambient and Pervasive Interactions - 5th International Conference, DAPI 2017 Held as Part of HCI International 2017, Proceedings (Lecture Notes in Computer Science (including subseries Lecture Notes in Artificial Intelligence and Lecture Notes in Bioinformatics)), Norbert Streitz and Panos Markopoulos (Eds.). Springer Verlag, Vancouver, Canada, 472-481. https://doi.org/10.1007/978-3-319-58697-7 35

[92] Petra Sundström, Elsa Vaara, Jordi Solsona, Niklas Wirström, Marcus Lundén, Jarmo Laaksolhati, Annika Waern, and Kristina Höök. 2011. Experiential arti facts as a design method for somaesthetic service development. In Proceedings of the 2011 ACM symposium on The role of design in UbiComp research \& practice - RDURP '11. ACM Press, Beijing, China, 33. https://doi.org/10.1145/2030031. 2030041
[93] Auditya Purwandini Sutarto, Muhammad Nubli Abdul Wahab, and Nora Mat Zin. 2012. Resonant Breathing Biofeedback Training for Stress Reduction Among Manufacturing Operators. International fournal of Occupational Safety and Ergonomics 18, 4 (2012), 549-561. https://doi.org/10.1080/10803548.2012.11076959 PMID: 23294659.

[94] Faisal Taher, John Hardy, Abhijit Karnik, Christian Weichel, Yvonne Jansen, Kasper Hornbæk, and Jason Alexander. 2015. Exploring Interactions with Physically Dynamic Bar Charts. In Proceedings of the 33rd Annual ACM Conference on Human Factors in Computing Systems (Seoul, Republic of Korea) (CHI '15). Association for Computing Machinery, New York, NY, USA, 3237-3246. https://doi.org/10.1145/2702123.2702604

[95] James Keng Soon Teh, Adrian David Cheok, Yongsoon Choi, Charith Lasantha Fernando, Roshan Lalintha Peiris, and Owen Noel Newton Fernando. 2009. Huggy pajama: a parent and child hugging communication system. In Proceedings of the 8th International Conference on Interaction Design and Children - IDC '09. ACM Press, Como, Italy, 290. https://doi.org/10.1145/1551788.1551861

[96] Kiyoshi Tomimatsu and Xiaotian Sun. 2016. Breathing shelter-Relieve citizen pressure with the breath control. ADADA international conference 2016 proceedings 15 (Nov. 2016), 3-7.

[97] Muhammad Umair, Corina Sas, and Muhammad Hamza Latif. 2019. Towards Affective Chronometry: Exploring Smart Materials and Actuators for Real-time Representations of Changes in Arousal. In Proceedings of the 2019 on Designing Interactive Systems Conference - DIS '19. ACM Press, San Diego, CA, USA, 14791494. https://doi.org/10.1145/3322276.3322367

[98] Jan Van Dixhoorn. 2007. Whole body breathing. Principles and practices of stress management 36, 3 (2007), 291-332.

[99] Marieke van Rooij, Adam Lobel, Owen Harris, Niki Smit, and Isabela Granic. 2016. DEEP: A Biofeedback Virtual Reality Game for Children At-risk for Anxiety. In Proceedings of the 2016 CHI Conference Extended Abstracts on Human Factors in Computing Systems - CHI EA '16. ACM Press, San Jose, California, USA, 1989-1997. https://doi.org/10.1145/2851581.2892452

[100] Evgeny G Vaschillo, Bronya Vaschillo, and Paul M Lehrer. 2006. Characteristics of resonance in heart rate variability stimulated by biofeedback. Applied psychophysiology and biofeedback 31, 2 (2006), 129-142.

[101] Cati Vaucelle, Leonardo Bonanni, and Hiroshi Ishii. 2009. Design of haptic interfaces for therapy. In Proceedings of the 27th international conference on Human factors in computing systems - CHI 09. ACM Press, Boston, MA, USA, 467. https://doi.org/10.1145/1518701.1518776

[102] Jay Vidyarthi, Bernhard E. Riecke, and Diane Gromala. 2012. Sonic Cradle: designing for an immersive experience of meditation by connecting respiration to music. In Proceedings of the Designing Interactive Systems Conference on - DIS '12. ACM Press, Newcastle Upon Tyne, United Kingdom, 408. https: //doi.org/10.1145/2317956.2318017

[103] Kanit Wongsuphasawat, Alex Gamburg, and Neema Moraveji. 2012. You Can't Force Calm: Designing and Evaluating Respiratory Regulating Interfaces for Calming Technology. In Adjunct Proceedings of the 25th Annual ACM Symposium on User Interface Software and Technology (Cambridge, Massachusetts, USA) (UIST Adjunct Proceedings '12). Association for Computing Machinery, New York, NY, USA, 69-70. https://doi.org/10.1145/2380296.2380326

[104] Libby Worth. 2015. Symmetry and asymmetry in dance and movement training using the Feldenkrais Method ${ }^{\circledR}$. Theatre, Dance and Performance Training 6, 2 (May 2015), 130-144. https://doi.org/10.1080/19443927.2015.1049708

[105] Bin Yu, Loe Feijs, Mathias Funk, and Jun Hu. 2015. Breathe with Touch: A Tactile Interface for Breathing Assistance System. In Human-Computer Interaction - INTERACT 2015, Julio Abascal, Simone Barbosa, Mirko Fetter, Tom Gross, Philippe Palanque, and Marco Winckler (Eds.). Vol. 9298. Springer International Publishing, Cham, 45-52. https://doi.org/10.1007/978-3-319-22698-9_4 Series Title: Lecture Notes in Computer Science.

[106] Semir Zeki. 2013. Clive Bell's "Significant Form" and the neurobiology of aesthetics. Frontiers in Human Neuroscience 7 (Nov. 2013), 1-14. https://doi. org/10.3389/fnhum.2013.00730 